\title{
Potential of Voronoi Diagram for the Conserved Remapping of Precipitation
}

\author{
KI-Hwan Kim, Eun-HeE LeE, AND Song-You Hong \\ Korea Institute of Atmospheric Prediction Systems, Seoul, South Korea
}

(Manuscript received 20 November 2017, in final form 21 May 2018)

\begin{abstract}
This study considers a remapping algorithm between irregularly distributed observations and grid points based on Voronoi diagrams and examines its potential for verification of precipitation forecasts. We propose a new remapping method using Voronoi diagrams to apply conservative area-weighted remapping on grid data to station points, describing a representative area for each station point. Conservative remapping is applied to interpolate daily precipitation data between grid and station points over South Korea. The proposed method shows significant differences from bilinear interpolation, which has been widely used in modeling communities, for local maximum and mean in a given domain. It is also shown that different interpolation methods have an impact on verification results of precipitation forecast from a numerical weather prediction model against station observations. It is suggested that the proposed method has potential to be used for verifying precipitation forecasts at in situ observation points, with its conservativeness and capability to be applied in any remapping direction between grids and stations.
\end{abstract}

\section{Introduction}

Modeling and forecasting precipitation is very important for a wide range of space and time scales (Michaelides 2008). The ability of a model to predict rainfall accurately is often used as an indicator of overall model predictability, particularly for operational numerical weather prediction (NWP) (Olson et al. 1995; Mesinger 1996; Du et al. 1997). Therefore, verifying quantitative precipitation forecasts (QPFs) to assess NWP model accuracy is of great interest (Golding 2000; McBride and Ebert 2000; Ebert et al. 2003; Rossa et al. 2008). One of the issues in QPFs might be spatial matching between forecast product and reference data, both for model-oriented verification (verifying on the model grid points) and useroriented verification (verifying on the observation points) (Tustison et al. 2001; Cherubini et al. 2002; Accadia et al. 2003). Lots of studies have investigated precipitation prediction sensitivity to interpolation. For example, among a few existing interpolation methods, bilinear interpolation tends to smooth the maximum and increase the minimum of the precipitation field over the grid, with consequential impacts on precipitation skill scores, such as equitable

Corresponding author: Eun-Hee Lee, eh.lee@kiaps.org threat score and Heidke skill score, compared with nearest-neighbor area-averaging methods (Accadia et al. 2003). It is also pointed out that bilinear interpolation does not conserve the total amount of precipitation satisfactorily (McBride and Ebert 2000; Accadia et al. 2003).

Precipitation, which is measured by amount per unit area, represents an important water source from the atmosphere to the surface. Therefore, conservative interpolation or remapping must be considered in hydrology. Area-weighted interpolation between gridded data (e.g., nearest-neighbor area averaging; McBride and Ebert 2000; Accadia et al. 2003) is conservative, provided all representative areas are included and do not overlap. However, for remapping gridded data to irregularly distributed station points, assigning relevant and representative station point areas is problematic. This often occurs when verifying precipitation forecasts for operational NWP models against rain-gauge observation data. Pointwise in situ precipitation measurement is often considered the reference value for validating all other precipitation products or forecasts. Therefore, it might be practical to verify precipitation products using rain-gauge observations by considering their conservativeness of hydrological budget, although we need to assume the representativeness of the point observation for the assigned area. 
Conservative remapping has focused on maintaining the model energy and water budgets, and it has been developed and applied to conserve state variables and heat and water fluxes transferred between source and target grids. This method was originally introduced by Dukowicz and Kodis (1987), and its application to spherical coordinates was developed by Jones (1999). In addition to these methods, Geometrically Exact Conservative Remapping (GECoRe) (Ullrich et al. 2009) has been introduced to calculate area integration exactly on the sphere. More recently, Kritsikis et al. (2017) also suggest a methodology applicable to remapping between general spherical grids. One of the main concerns of these approaches is how to remap exactly on geometric point of view. However, there is some limitation to grid-to-station interpolation approaches (e.g., GECoRe). Though the method addressed by Kritsikis et al. (2017) has more flexibility, defining representative areas for irregularly located observation stations remains an open issue.

This study proposes a conservative remapping method based on Voronoi diagrams to interpolate precipitation between the model grid and observation station points. Voronoi tessellation is used to generate the grid cells represented by the irregularly distributed observation points. Dirichlet (1850) initially proposed Voronoi tessellation, which was finally established by Voronoi (1908) and has been subsequently widely used in various fields, such as biology (Bock et al. 2010), Thiessen polygons in meteorology (Thiessen 1911), and Wigner-Seitz cells in solid state physics (Wigner and Seitz 1933). Voronoi diagrams arise in nature in various situations, and their simple and intuitive applicability makes them a powerful tool to solve spatial partition problems (Aurenhammer 1991). Therefore, we propose Voronoi tessellation to generate grid cells represented by irregularly distributed observation points, which are required for conservative remapping.

The proposed method is applied to remap daily precipitation product obtained from the TRMM Multisatellite Precipitation Analysis (TMPA) (Huffman et al. 2007) to automatic weather station (AWS) points over South Korea. We compare the proposed method characteristics, including conservativeness, with the bilinear method, which is commonly used for grid-to-station interpolation. To examine the impact of the proposed method on verification skill scores, precipitation forecasts from an NWP model are verified against AWS observations and compared with results of the different interpolation method.
Section 2 introduces the remapping method, and evaluation results are presented in section 3. Section 4 examines the impact of the proposed conservative remapping method on NWP verification. Section 5 summarizes and concludes the paper.

\section{Conservative remapping using Voronoi diagrams}

For precipitation $F$, which is defined as the continuous function over the domain $\Omega$, the total amount of precipitation (Total Precip.) over a given domain is defined by area integration and can be expressed as

$$
\text { Total Precip. }=\int F(\lambda, \theta) d \Omega \text {. }
$$

Since the observation points and numerical model grid are discretized, we may assume that the value at a given grid point is the mean for the area surrounding the point and define the total precipitation at an observation station or grid point as

$$
\text { Total Precip. }=\sum_{i} f_{i} A_{i},
$$

where $f_{i}$ and $A_{\mathrm{i}}$ are the value and area, respectively, at grid point $i$.

If a method conserves area integration on the grid cell before and after remapping, that is,

$$
\int F_{\text {target }} d \Omega=\int F_{\text {source }} d \Omega
$$

it is called a conservative remapping.

To achieve conservative remapping, grid cells should be defined as an area represented by a grid point, even for the irregularly located stations. To solve this, we chose a method to define the cells as the region nearest to each station using a Voronoi diagram. We employed this to define the grid cell $A_{i}$ and used Voronoi tessellation to divide the space into a number of regions, with each grid point treated as a corresponding region. Voronoi tessellation is constructed by bisecting lines with adjacent points (Aurenhammer 1991) in two steps:

1) Delaunay triangles are constructed connecting the points to all of their neighbors without crossing.

2) The Voronoi diagram is constructed by connecting the center circumcircles of the Delaunay triangles.

Figure 1 compares conservative remapping using Voronoi diagrams with bilinear interpolation. Bilinear 
(a) Bilinear interpolation

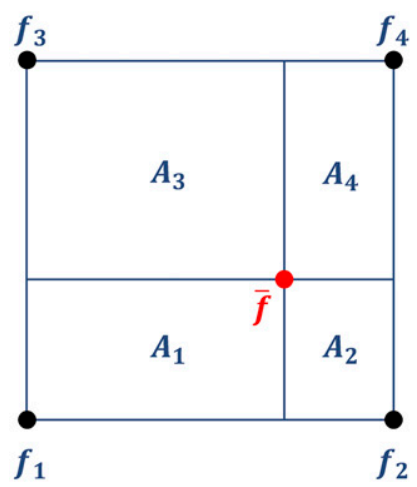

(b) Conservative remapping

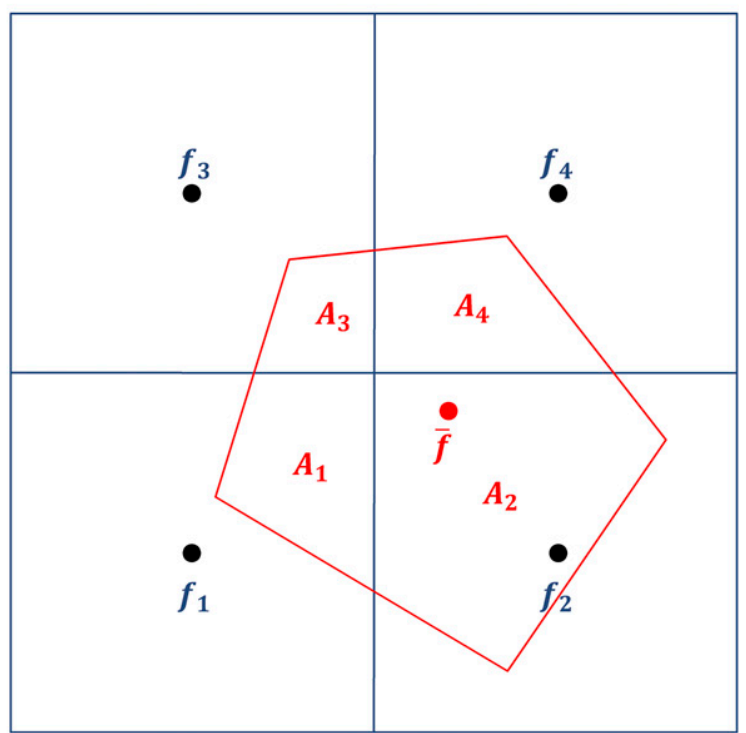

FIG. 1. Schematic diagram of bilinear interpolation and conservative remapping, where $f_{1-4}$ is the value at the source grid, $A_{1-4}$ is the representative area of individual point $f$ used for the interpolation, and $\bar{f}$ is the interpolated value at the target point.

interpolation is performed by two linear interpolations (Fig. 1a) in separate directions. If the values at grid points are $f_{1}, f_{2}, f_{3}$, and $f_{4}$, as shown in Fig. 1 a, the interpolated value can be expressed as

$$
\bar{f}=\frac{1}{A}\left(A_{4} f_{1}+A_{3} f_{2}+A_{2} f_{3}+A_{1} f_{4}\right)
$$

where $A_{i}$ is representative area of an individual point, and $A=A_{1}+A_{2}+A_{3}+A_{4}$. For bilinear interpolation between regular grid and irregular measurement stations, total precipitation cannot be measured by integrating discretized $f$ and area $\omega$ because $\omega$ is not defined.

Grid cells with different shapes can be defined when remapping using Voronoi diagrams, depending on the gridpoint distribution, as shown in Fig. 1b. For example, source grid cells can be defined as rectangular, whereas target grid cells are pentagons. The interpolated value can be expressed as

$$
\bar{f}=\frac{1}{A}\left(A_{1} f_{1}+A_{2} f_{2}+A_{3} f_{3}+A_{4} f_{4}+\cdots\right),
$$

where $A=A_{1}+A_{2}+A_{3}+A_{4}+\ldots$. This remapping becomes the first-order conservative property (i.e., areaweighted interpolation) when the gridcell domain values are constant, and it is apparently conservative.
Figure 2 shows the process to define target grid cells for observation stations using Voronoi tessellation, where the sample stations are the AWS observation sites over South Korea. There are 670 observation stations, with relatively high spatial density in Seoul and adjacent metropolitan areas (Fig. 2a). We first constructed the Voronoi diagram from the site locations' information, using the Python scipy.spatial.Voronoi module for Voronoi tessellation (SciPy 2017), as shown in Fig. 2b. It appears that the area of a cell varies depending highly on the spatial density of stations; it is larger where the observation station density is relatively low, particularly for coastal and surrounding regions.

Therefore, pseudostations were generated as shown in Fig. 2c (red dots) to smooth spatial density, where pseudostation distances were determined by half of the mean distance of real stations. The Voronoi diagram was then regenerated, including real and pseudostations, as shown in Fig. 2d. The resulting Voronoi diagram demonstrates that grid cells represent areas with comparable sizes. Blue grid cells designate sites including actual observation stations, whereas red grid cells indicate no station.

\section{Evaluation of the conservative remapping}

Experiments are conducted by using gridded satellite precipitation data for a heavy rain case over the Korean 
(a) AWS locations

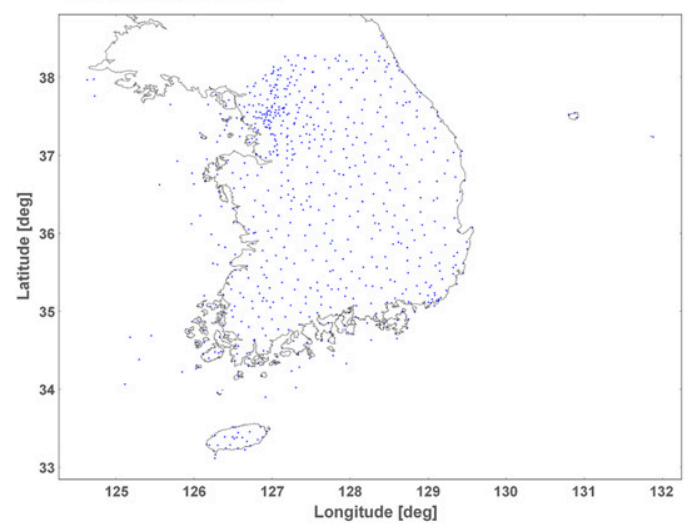

(c) Append pseudo stations

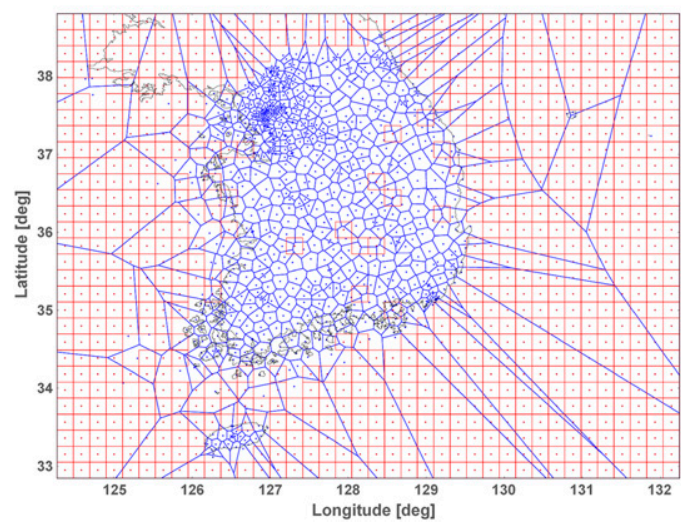

(b) First Voronoi tessellation

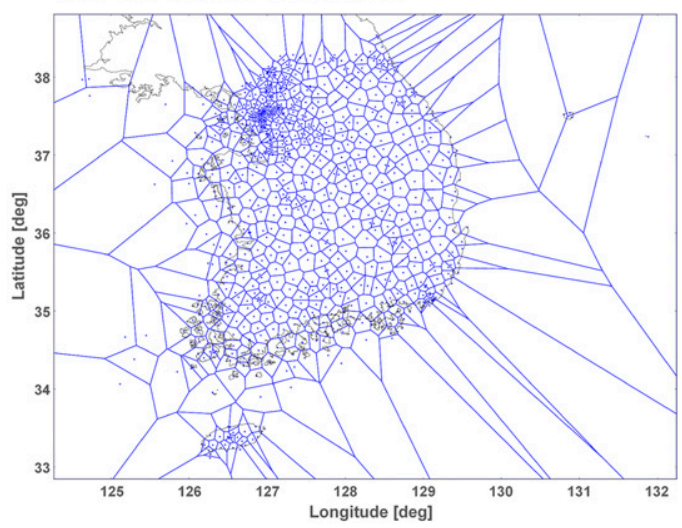

(d) Second Voronoi tessellation

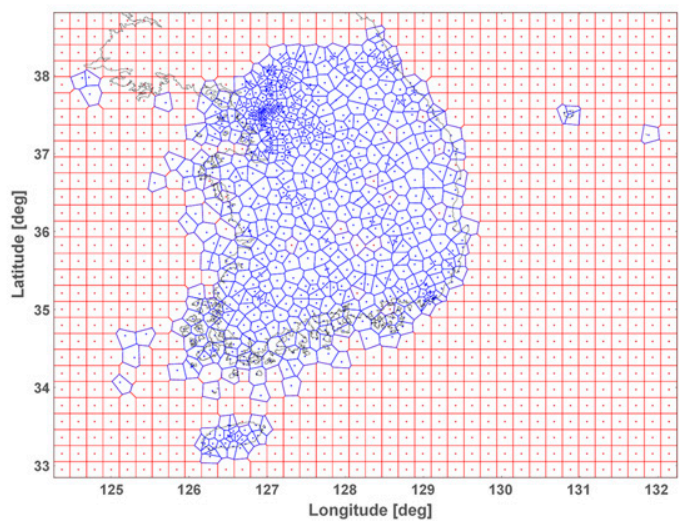

FIG. 2. Process of Voronoi tessellation for station sites: (a) distribution of 670 AWS stations over South Korea, (b) the first Voronoi tessellation using only AWS locations, (c) appended pseudostations (red dots) to mask regions not covered by AWS, and (d) the second Voronoi tessellation with both real (blue dots) and pseudostations (red dots).

Peninsula for evaluating the proposed conservative remapping method with the Voronoi diagram. The 24-h accumulated precipitation product from TMPA with $0.25^{\circ}$ horizontal resolution was remapped to AWS observation sites for a heavy rainfall case observed at 0000 UTC 26 July 2011. Jang and Hong (2014) presented a synoptic overview of precipitation characteristics of this event, along with global model predictability.

Figure 3a shows the precipitation distribution obtained from TMPA precipitation for the case day, and Fig. $3 b$ shows AWS observed daily precipitation for the same day, with AWS site representative areas from the Voronoi diagram shown in Fig. 2d. Slash-shaded areas represent undefined values due to pseudopoints in the remapping process (Figs. 2c,d).

The evaluation is based on the comparison with the bilinear interpolation method, which is commonly employed, particularly focusing on the following questions:
1) Which method is better for conserving the total amount of precipitation?

2) Which method is better for representing local maximum and minimum values?

3) Which method shows higher remapping accuracy between the original data and remapped data?

To answer the questions, the evaluation is performed in two ways. First, total and maximum amounts of precipitation were compared between source grid and remapped station point data using one-way remapping (grid $\rightarrow$ station). Second, relative differences were examined on source grid space by comparing original and reverse remapped data from observation points (grid $\rightarrow$ station $\rightarrow$ grid).

In the conservative remapping using the Voronoi meshes, grid-to-station remapping and the reverse remapping from station to grid can be specified in the same way, since the weighted area is calculated by 
(a) TMPA observations (26 th July 2011 UTC)

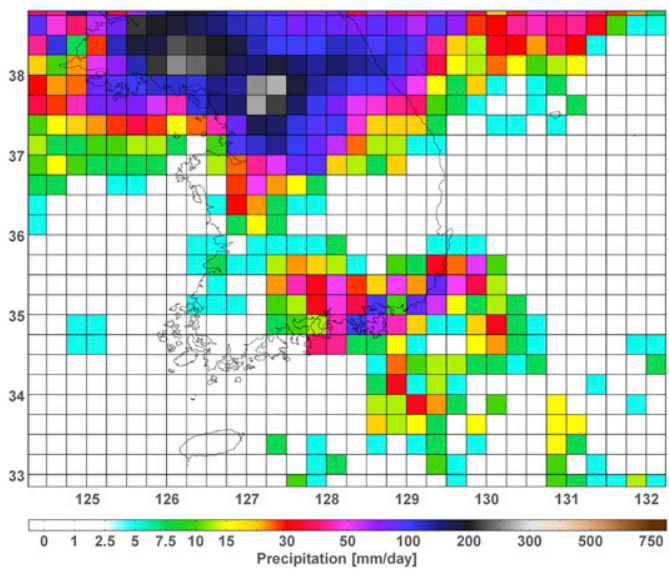

(b) AWS observations (26 th July 2011 UTC)

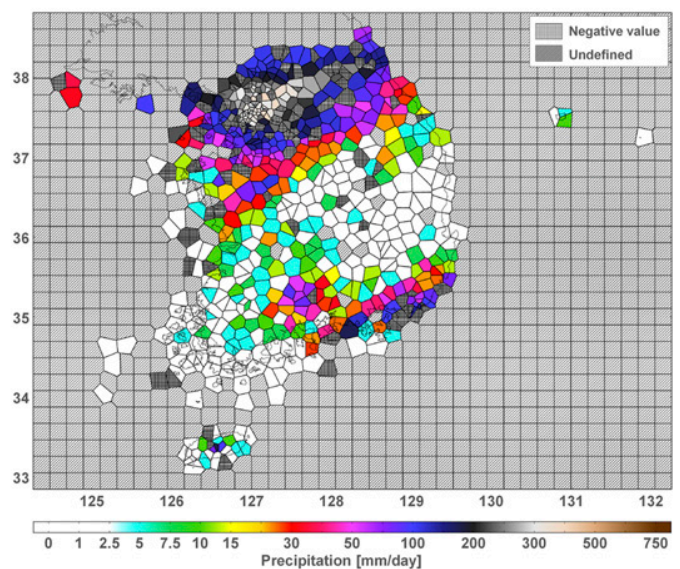

FIG. 3. Daily precipitation observations at 0000 UTC 26 Jul 2011 obtained from TMPA and AWS: (a) TMPA original $\left(0.25^{\circ}\right.$ grid $)$ and (b) AWS precipitation on Voronoi cells.

general ways to determine polygon areas. However, a different method was required to obtained gridded data from bilinear interpolated station data. Therefore, the Cressman scheme (Cressman 1959) was applied, a commonly employed objective analysis from station to grid. The Cressman scheme uses inverse distance weight within a prescribed radius of influence, whose weighting is described as

$$
\begin{array}{ll}
w_{j}=\frac{\left(d^{2}-r^{2}\right)}{\left(d^{2}+r^{2}\right)}, & r<d, \\
w_{j}=0, & r \geq d,
\end{array}
$$

where $r$ is the distance from the grid point to the observation, and $d$ is radius of influence. The oacres function in the Grid Analysis and Display System (GrADS) (Doty 1995) was used for Cressman interpolation. In the defaults, the radii of influence are $10,7,4,2$, and 1 in grid units. Multiple passes are made through the grid with increasingly smaller radii of influence. At each pass, a new value is calculated for each grid point based on a correction factor that is determined by looking at each station within the radius.

Figure 4 compares the proposed method with conservative remapping and other methods: Fig. 4a shows remapped daily precipitation converted from the original TMPA grid-to-station sites using conservative remapping, and Fig. 4b shows the difference from the results using bilinear interpolation outcomes. It appears that there is a noticeable difference between the interpolated data with the discrepancy dependent on station density. As is well known, bilinear interpolation affects the precipitation field by smoothing the maxima and smearing and increasing the minima, and it is especially significant for remapping at a higher resolution. Thus, the difference between two results becomes larger when the target cells are highly resolvable (i.e., smaller). It shows that discrepancy increases for regions with higher station density and, conversely, is small for regions with lower station density. For example, the difference is very small, even for heavy rainfall regions in the northern part of the domain. Figure $4 \mathrm{c}$ shows the reverse remapped data on the original source grid space with $0.25^{\circ}$ resolution from remapped data at station points (Fig. 4a) using conservative remapping, and Fig. 4d shows the difference from the Cressman method. The Cressman method produces additional grid-to-station differences (Fig. 4b) over the domain.

The quantitative evaluation results of the remapping method with a Voronoi diagram that is developed in this study are given by comparing results with a conventional method using bilinear interpolation and Cressman analysis, which are given in Tables 1 and 2. It is apparent that the total amount of precipitation is conserved using the new remapping method both for grid $\rightarrow$ station remapping and grid $\rightarrow$ station $\rightarrow$ grid remapping, whereas the corresponding amount is reduced from $1109.29 \times 10^{3}$ to $1104.09 \times 10^{3}$ and $1104.01 \times 10^{3} \mathrm{~mm} \mathrm{day}^{-1}$ using the conventional technique. Although the maximum decreases for both techniques, the reduction is particularly smaller for the proposed Voronoi diagrambased remapping. The proposed method captures extrema on the mesh better since maximum precipitation occurs where station density is higher than the source grid resolution (Fig. 4a). Bilinear interpolation tends to smooth precipitation maxima 

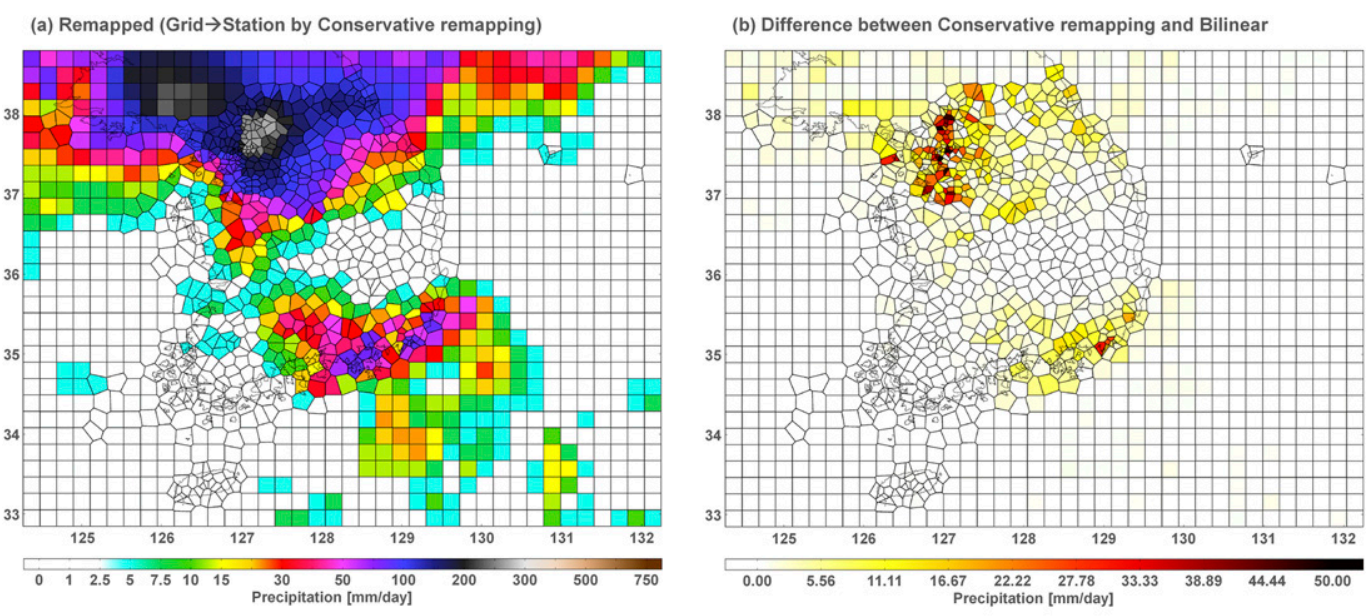

(c) Remapped (Grid $\rightarrow$ Station $\rightarrow$ Grid by Conservative remapping)
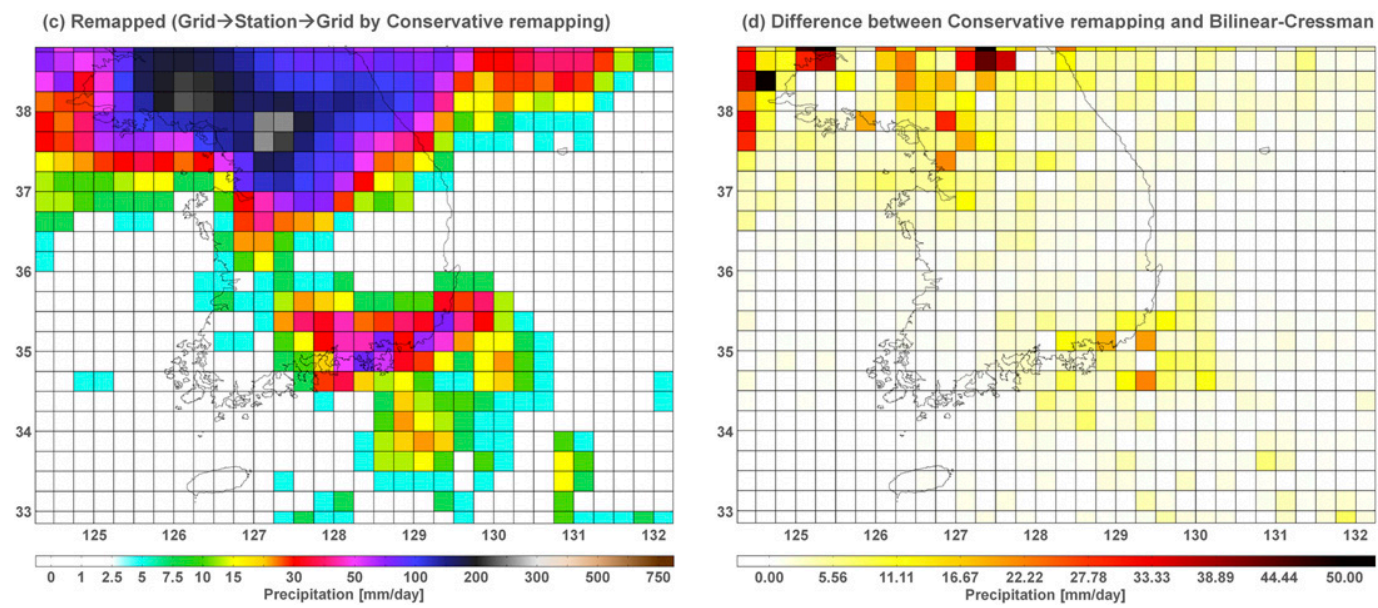

FIG. 4. Comparison of remapping schemes for a heavy rainfall event over South Korea on 26 Jul 2011: (a) TMPA precipitation remapped on station cells, (b) precipitation difference between the proposed conservative remapping and bilinear interpolation, (c) reverse remapped precipitation from remapped station point to original grid (grid $\rightarrow$ station $\rightarrow$ grid), and (d) precipitation difference from results with bilinear interpolation and Cressman method.

and increase minima over the grid, particularly when the target grid has higher resolution. In the case of the proposed remapping, it will capture the extrema exactly if a target Voronoi cell fits within the source grid.

Table 2 compares relative differences between original grid and reverse remapped grid data. The relative error norms $L_{1}, L_{2}$, and $L_{\infty}$ are defined as

$$
\begin{array}{r}
L_{1}=\frac{\sum_{i}\left|\left(x_{1}-x_{0}\right)_{i}\right|}{\sum_{i}\left|\left(x_{0}\right)_{i}\right|}, \\
L_{2}=\frac{\sqrt{\sum_{i}\left(x_{1}-x_{0}\right)_{i}^{2}}}{\sqrt{\sum_{i}\left(x_{0}\right)_{i}^{2}}},
\end{array}
$$

and

$$
L_{\infty}=\frac{\max \left[\left|\left(x_{1}-x_{0}\right)_{i}\right|\right]}{\max \left[\left|\left(x_{0}\right)_{i}\right|\right]},
$$

where $x_{0}$ and $x_{1}$ are original and remapped values at grid point $i$. It is obvious that the error for Voronoi-based conservative remapping is smaller than for conventional interpolation.

\section{Impact on NWP verification}

We examined the effect of remapping methods on statistical verification of daily precipitation prediction products. Medium-range forecast experiments for a 1-month period were performed using an NWP model. A global model with a nonhydrostatic dynamical core 
TABLE 1. Comparison of conservation of total precipitation and decrease of maximum precipitation.

\begin{tabular}{llcrr}
\hline \hline & \multicolumn{1}{c}{ Methods } & TMPA & Grid $\rightarrow$ station & Grid $\rightarrow$ station $\rightarrow$ grid \\
\hline Total precipitation $\left(\times 10^{3} \mathrm{~mm} \mathrm{day}^{-1}\right)$ & Bilinear and Cressman & 1109.29 & 1104.09 & 1104.01 \\
& Conservative remapping & & 1109.29 & 1109.29 \\
Maximum precipitation $\left(\mathrm{mm} \mathrm{day}^{-1}\right)$ & Bilinear and Cressman & 263.83 & 251.94 & 238.68 \\
& Conservative remapping & & 263.73 & 248.58 \\
\hline
\end{tabular}

on a cubed sphere grid developed by Choi and Hong (2016) with the full physics package (Hong et al. 2018) was employed for the simulation. Horizontal resolution is approximately $50 \mathrm{~km}$ (NE60NP4), and the hybrid sigma coordinate is used for the 50 vertical layers up to $0.3 \mathrm{hPa}$. Seventy-two-hour simulations were performed every day at 0000 UTC during July 2013 using initial data from the Global Forecast System (GFS) at the National Centers for Environmental Prediction (NCEP). Daily SST and sea ice information were also provided by NCEP GFS.

To verify the predicted precipitation against surface observation, cubed sphere results were interpolated or remapped to the observation points using the proposed Voronoi-based and conventional bilinear remapping methods. Daily precipitation forecast accuracy was quantitatively verified by assessing conditional detection, based on a $2 \times 2$ contingency table, as shown in Table 3. The total number of hits $H$, misses $M$, false alarms $F$, and correct negatives (CN) were obtained for given thresholds and categorical statistics derived.

Figure 5 shows frequency bias and equitable threat score (ETS) for precipitation thresholds $0.5,1,5,10,15$, 20, and $25 \mathrm{~mm} \mathrm{day}^{-1}$,

$$
\text { Frequency bias }=\frac{H+F}{H+M} \text {, }
$$

and

$$
\mathrm{ETS}=\frac{H-a_{r}}{H+M+F-a_{r}}, \quad a_{r}=\frac{(H+F)(H+M)}{H+M+F+\mathrm{CN}}
$$

It appears that differences in NWP skill between the two interpolation methods are distinct. The bilinear method shows enhanced frequency bias for light precipitation thresholds and reduced frequency bias for heavy precipitation categories (Fig. 5a). The impact of the interpolation method is significant for most precipitation thresholds, except for 15 and $20 \mathrm{~mm} \mathrm{day}^{-1}$. It is found that the ETS skill scores between the two interpolation methods also exhibit discrepancies for overall threshold ranges. Differences in the ETS score are significant for the very light precipitation threshold of $0.5 \mathrm{~mm}$ and between $5.0-15 \mathrm{~mm}$ day $^{-1}$. For example, the proposed remapping method shows a reduced skill score by $2 \%-4 \%$ for $5.0-15 \mathrm{~mm}^{2}$ day $^{-1}$ thresholds, compared with that from the results using the bilinear method (Fig. 5b).

To clarify the reason for the differences in precipitation forecasting skill between the two interpolation methods, the distribution of precipitation frequencies is compared in Fig. 6. It is seen that the bilinear interpolation method tends to increase light precipitation while reducing the number of heavy precipitation activities, compared to the conservative remapping. This is the reason why the statistics obtained by bilinear interpolation shows a higher bias with a light precipitation threshold but a reduced frequency bias with heavy precipitation greater than $20 \mathrm{~mm} \mathrm{day}^{-1}$, as shown in Fig. 5.

The difference in the ETS scores between the two remapping methods can be explained by examining the relationship between the bias and ETS skill scores. For light precipitation events, an increase in overprediction (bias $>1$ ) using bilinear interpolation reduces the ETS score by raising the frequency of false alarm events. On the contrary, overprediction enhances the ETS score for the threshold range of 5-15 $\mathrm{mm}$ turns out to be sensitive to missed events. This behavior in the ETS score related to the bias score is explained by its dependency on event size, as mentioned by Baldwin and Kain (2006). For rarely observed events, accuracy measures can be

TABLE 2. Comparison of relative differences between the original and remapped TMPA data.

\begin{tabular}{cccc}
\hline \hline & & $\begin{array}{c}\text { Grid } \rightarrow \text { station } \rightarrow \\
\text { grid }\end{array}$ \\
\hline Standard & Methods & & 0.2007 \\
errors & Bilinear and & $L_{1}$ & 0.2203 \\
& Cressman & $L_{2}$ & 0.4067 \\
& & $L_{\infty}$ & 0.1314 \\
& Conservative & $L_{1}$ & 0.1453 \\
& remapping & $L_{2}$ & 0.3588 \\
\hline
\end{tabular}


TABLE 3. Contingency table of possible precipitation events for a selected threshold.

\begin{tabular}{llll}
\hline \hline & & \multicolumn{2}{c}{$\begin{array}{c}\text { Precipitation } \\
\text { observed }\end{array}$} \\
\cline { 3 - 4 } & & Yes & No \\
\hline Precipitation forecast & Yes & $H$ & $F$ \\
& No & $M$ & CN \\
\hline
\end{tabular}

maximized for a bias greater than 1 since it is more sensitive to missed events.

\section{Summary and discussion}

This study proposed a conservative remapping method based on Voronoi diagrams to interpolate meteorological data between grids and stations. The proposed technique was applied to the precipitation data of Korea and compared with conventional interpolation. The proposed method is shown to be better than bilinear interpolation with Cressman's weighting average in the aspect of conserving domain-averaged total and maximum precipitation. Remapping accuracy of the proposed method is also higher than bilinear interpolation. Therefore, the Voronoi-based method could be useful for verifying modeled precipitation against in situ observation data. In contrast to the conventional approach, the proposed method can be applied in any remapping direction between grids and stations, and the Voronoi diagram proved a useful tool for visualization of station data.

This study assumed that a single point measurement could represent as an assigned Voronoi cell (i.e., the spatial average for a given cell). However, spatiotemporal behavior of precipitation within a given cell depends on many factors, including terrain geographic characteristics and the precipitation measurement system (convective, orographic, or frontal). Therefore, considering only station density could be a limitation of the proposed approach and should be investigated in future studies. Many low-density rural stations were probably poorly representative of precipitation over the full Voronoi cell area. Nevertheless, information from the assigned Voronoi cell allowed visualizing station and density modulation by appending
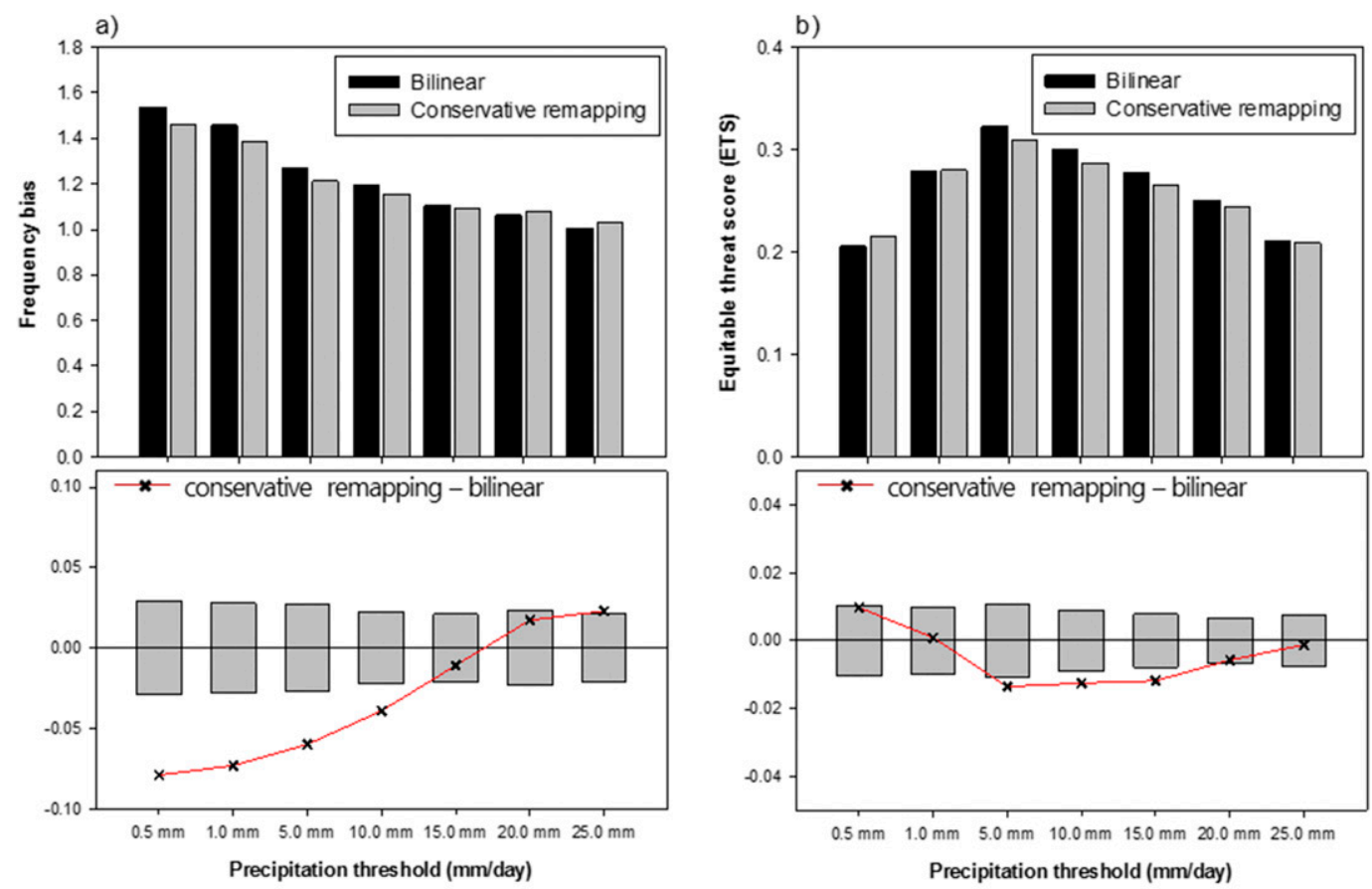

Difference outside of the shaded bars are $95 \%$ significant based on 10000 Monte Carlo Tests

FIG. 5. (a) Frequency bias and (b) ETS for daily precipitation thresholds during 72-h lead time verified against precipitation observations postprocessed with bilinear and proposed Voronoi-based conservative remapping methods. Differences between two methods are given below, with $95 \%$ significant test results presented at the bottom. 
(a) Frequency distribution of precipitation prediction
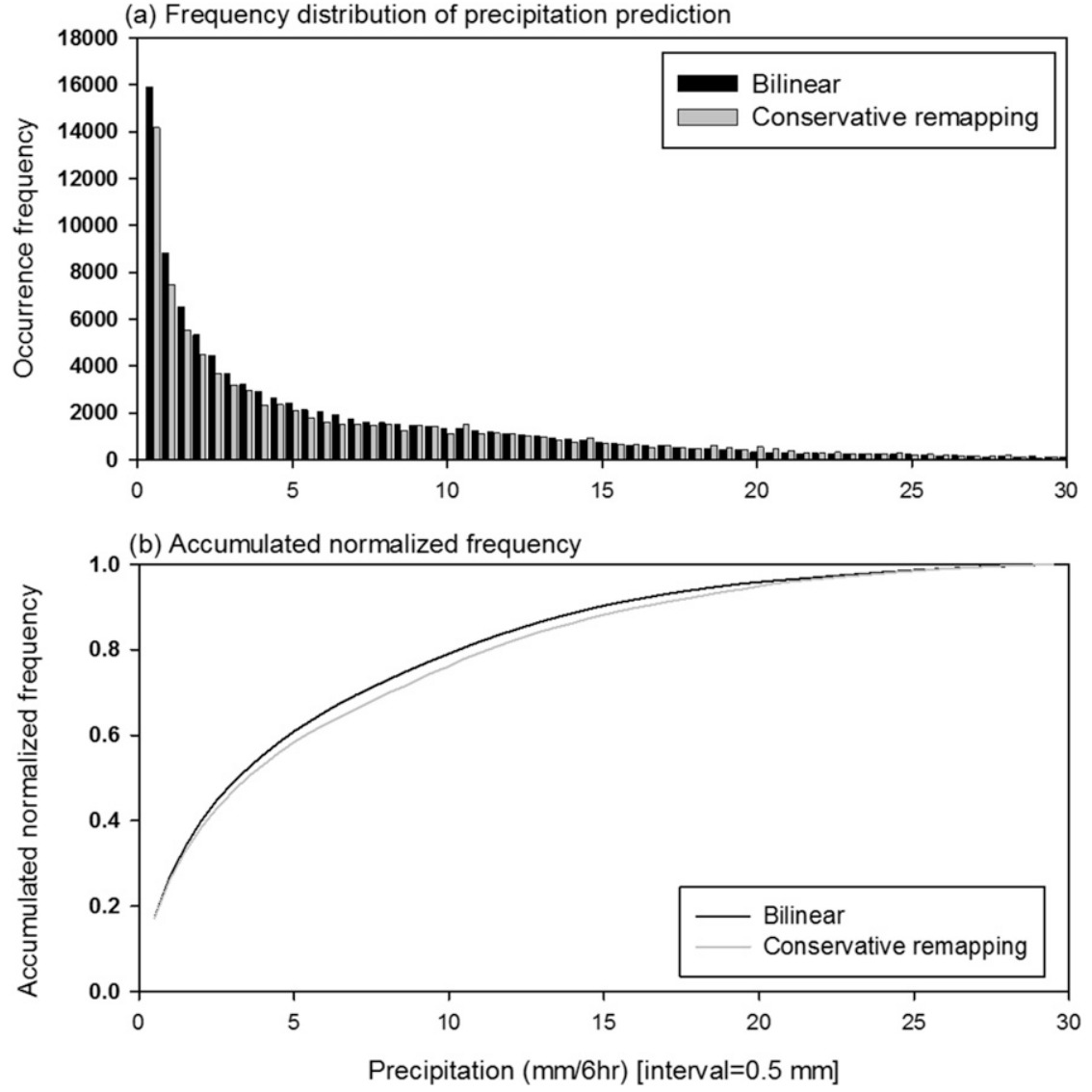

FIG. 6. (a) Occurrence frequency and (b) accumulated normalized frequency distributions of 6-hourly accumulated precipitation prediction interpolated on the AWS station points using the bilinear method and conservative remapping. Normalized accumulation was conducted in the prediction range of $0.5-30 \mathrm{~mm}$.

pseudostation points, which may provide expanded application.

In this study, we evaluated the proposed remapping method for local domains. However, the application of the remapping method on a sphere is straightforward since Voronoi tessellation and polygon weighting can be realized in 3D space with coordinate information for the points. For example, Na et al. (2002) performed Voronoi tessellation on a sphere directly. Further, the proposed method has the potential to be applied in any remapping between grids and stations by virtue of its conservativeness.

Acknowledgments. This work has been carried out through the R\&D project on the development of global numerical weather prediction systems of the Korea Institute of Atmospheric Prediction Systems (KIAPS), funded by the Korea Meteorological Administration (KMA). The authors thank Dr. Sanghun Lee for processing the observation data and helpful discussions.

\section{REFERENCES}

Accadia, C., S. Mariani, M. Casaioli, A. Lavagnini, and A. Speranza, 2003: Sensitivity of precipitation forecast skill scores to bilinear interpolation and a simple nearestneighbor average method on high-resolution verification grids. Wea. Forecasting, 18, 918-932, https://doi.org/10.1175/ 1520-0434(2003)018<0918:SOPFSS > 2.0.CO;2.

Aurenhammer, F., 1991: Voronoi diagrams-A survey of a fundamental geometric data structure. ACM Comput. Surv., 23, 345-405, https://doi.org/10.1145/116873.116880.

Baldwin, M. E., and J. S. Kain, 2006: Sensitivity of several performance measures to displacement error, bias, and event frequency. Wea. Forecasting, 21, 636-648, https://doi.org/10.1175/WAF933.1.

Bock, M., A. K. Tyagi, J.-U. Kreft, and W. Alt, 2010: Generalized Voronoi tessellation as a model of two-dimensional cell tissue dynamics. Bull. Math. Biol., 72, 1696-1731, https://doi.org/ 10.1007/s11538-009-9498-3.

Cherubini, T., A. Ghelli, and F. Lalaurette, 2002: Verification of precipitation forecasts over the Alpine region using a high-density observing network. Wea. Forecasting, 17, 238-249, https://doi.org/10.1175/1520-0434(2002)017<0238: VOPFOT $>2.0 . \mathrm{CO} ; 2$. 
Choi, S.-J., and S.-Y. Hong, 2016: A global non-hydrostatic dynamical core using the spectral element method on a cubedsphere grid. Asia-Pac. J. Atmos. Sci., 52 (3), 291-307.

Cressman, G. P., 1959: An operational objective analysis system. Mon. Wea. Rev., 87, 367-374, https://doi.org/10.1175/ 1520-0493(1959)087<0367:AOOAS > 2.0.CO;2.

Dirichlet, G. L., 1850: Über die Reduction der positiven quadratischen Formen mit drei unbestimmten ganzen Zahlen. J. Reine Angew. Math., 40, 209, https://doi.org/10.1515/crll.1850.40.209.

Doty, B., 1995: The Grid Analysis and Display System v1.5.1.12. Tech Rep., 145 pp., ftp://www.funceme.br/modelagem/echam46/ mar2013-2/GrADS_doc_1_5_1.pdf.

Du, J., S. L. Mullen, and F. Sanders, 1997: Short-range ensemble forecasting of quantitative precipitation. Mon. Wea. Rev., 125, 2427-2459, https://doi.org/10.1175/1520-0493(1997)125<2427: SREFOQ $>2.0 . \mathrm{CO} ; 2$.

Dukowicz, J. K., and J. W. Kodis, 1987: Accurate conservative remapping (rezoning) for arbitrary Lagrangian-Eulerian computations. SIAM J. Sci. Statist. Comput., 8, 305-321, https://doi.org/10.1137/0908037.

Ebert, E. E., U. Damrath, W. Wergen, and M. E. Baldwin, 2003: The WGNE assessment of short-term quantitative precipitation forecasts. Bull. Amer. Meteor. Soc., 84, 481-492, https://doi.org/10.1175/BAMS-84-4-481.

Golding, B. W., 2000: Quantitative precipitation forecasting in the UK. J. Hydrol., 239, 286-305, https://doi.org/10.1016/ S0022-1694(00)00354-1.

Hong, S.-Y., and Coauthors, 2018: The Korean Integrated Model (KIM) system for global weather forecasting. Asia-Pac. J. Atmos. Sci., in press.

Huffman, G. J., and Coauthors, 2007: The TRMM Multisatellite Precipitation Analysis (TMPA): Quasi-global, multiyear, combined-sensor precipitation estimates at fine scales. J. Hydrometeor., 8, 38-55, https://doi.org/10.1175/JHM560.1.

Jang, J., and S.-Y. Hong, 2014: Quantitative forecast experiment of a heavy rainfall event over Korea in a global model: Horizontal resolution versus lead time issues. Meteor. Atmos. Phys., 124, 113-127, https://doi.org/10.1007/s00703-014-0312-x.

Jones, P. W., 1999: First- and second-order conservative remapping schemes for grids in spherical coordinates. Mon. Wea. Rev., 127, 2204-2210, https://doi.org/10.1175/1520-0493(1999)127<2204: FASOCR $>2.0 . \mathrm{CO} ; 2$.

Kritsikis, E., M. Aechtner, Y. Meurdesoif, and T. Dubos, 2017: Conservative interpolation between general spherical meshes.
Geosci. Model Dev., 10, 425-431, https://doi.org/10.5194/ gmd-10-425-2017.

McBride, J. L., and E. E. Ebert, 2000: Verification of quantitative precipitation forecasts from operational numerical weather prediction models over Australia. Wea. Forecasting, 15, 103-121, https://doi.org/10.1175/1520-0434(2000)015<0103: VOQPFF $>2.0 . \mathrm{CO} ; 2$.

Mesinger, F., 1996: Improvements in quantitative precipitation forecasting with the Eta regional model at the National Centers for Environmental Prediction: The 48-km upgrade. Bull. Amer. Meteor. Soc., 77, 2637-2650, https://doi.org/10.1175/ 1520-0477(1996)077<2637:IIQPFW>2.0.CO;2.

Michaelides, S., 2008: Precipitation: Advances in Measurement, Estimation and Prediction. Springer, $540 \mathrm{pp}$.

Na, H.-S., C.-N. Lee, and O. Cheong, 2002: Voronoi diagrams on the sphere. Comput. Geom., 23, 183-194, https://doi.org/ 10.1016/S0925-7721(02)00077-9.

Olson, D. A., N. W. Junker, and B. Korty, 1995: Evaluation of 33 years of quantitative precipitation forecasting at the NMC. Wea. Forecasting, 10, 498-511, https://doi.org/10.1175/ 1520-0434(1995)010<0498:EOYOQP > 2.0.CO;2.

Rossa, A. M., P. Nurmi, and E. E. Ebert, 2008: Overview of methods for the verification of quantitative precipitation forecasts. Precipitation: Advances in Measurement, Estimation and Prediction, S. C. Michaelides, Ed., Springer, 418-450.

SciPy, 2017: SciPy reference guide v0.19.1. SciPy.org, accessed 12 March 2015, https://docs.scipy.org/doc/scipy/reference/.

Thiessen, A. H., 1911: Precipitation averages for large areas. Mon. Wea. Rev., 39, 1082-1089, https://doi.org/10.1175/1520-0493(1911) $39<1082$ b:PAFLA $>2.0$. CO;2.

Tustison, B., D. Harris, and E. Foufoula-Georgiou, 2001: Scale issues in verification of precipitation forecasts. J. Geophys. Res., 106, 11775-11 784, https://doi.org/10.1029/ 2001JD900066.

Ullrich, P. A., P. H. Lauritzen, and C. Jablonowski, 2009: Geometrically Exact Conservative Remapping (GECoRe): Regular latitude-longitude and cubed-sphere grids. Mon. Wea. Rev., 137, 1721-1741, https://doi.org/10.1175/ 2008MWR2817.1.

Voronoi, G., 1908: Nouvelles applications des paramètres continus à la théorie de formes quadratiques. J. Reine Angew. Math., 134, 198.

Wigner, E., and F. Seitz, 1933: On the constitution of metallic sodium. Phys. Rev., 43, 804, https://doi.org/10.1103/PhysRev.43.804. 